\title{
High accurite reconstruction of external ear by use low-cost 3D scanner
}

\author{
Mohammad H. Basheet* and Ziad T. Al-dahan \\ Biomedical Department of Engineering College, Al-Nahrain University, Baghdad, Iraq
}

\section{(C2017 ACCENTS}

\begin{abstract}
The 3D visualization recently played a significant role in biomedical application. It depends on computer environments, software and hardware tools that ease human-machine interaction. Biomedical visualization are considerably employed for the observation of the body's structure and in measuring of numerous properties of those structures, imaging strategies that utilized in medicine and biology are dependent on number of sources, including $x$-ray, magnetic resonance, electrons, laser, light, ,ultrasound. This study has presented a method of reconstruction $3 D$ external ear by utilized low cost $3 D$ sensor such as Microsoft Kinect with proposed reconstruction program to get high accuracy $3 D$ model of external ear. In 3D scanner of external ear, a Microsoft the Kinect version 1 for windows has been used along with a proposed software based on KinectFusion that designed in order to reconstruct external ear in with high accuracy in real-time. The results of external ear reconstruction showed good accuracy of ear details and measurement that related to reconstruction algorithm that yields good $3 D$ meshes of the external ear. This method with some modification can serve for manufacturing the low-cost accurate handled 3D ear scanner.
\end{abstract}

\section{Keywords}

3D Ear scanner, Ear reconstruction, Visualization, Low-cost 3D scanner.

\section{Introduction}

Computer vision is the science of getting details about the physical environment via images, will not focus on specific applications. However, its root base is in the field of what is known as artificial intelligence that aims at resembling intelligent human behaviour. A basic computer vision requires a camera, an interface of camera, as well as a computer.

A camera may be the basic sensing element. Basically, almost all cameras depend on the light property causes hole/electron pairs in a conducting material. Once a potential is utilized (in order to acquire the charge carriers), that charge could be detected as current. There are three typical cameras types, which are: charge coupled devices (CCDs), visions that are older (analog) technology, and currently, CMOS cameras. [1,2].

The most important goal of three- dimensional (3D) imaging is to present both qualitative and quantitative information with regards to a system or object from images acquired with multiple modalities such as: laser technique, SPECT, CT, MRI, and PET [3].

*Author for correspondence
3D scanners have become similar to cameras, it corresponding to cameras by has cone-like field of view (FOV), and also, they are able to just obtain information regarding surfaces which are not obscured. As the camera gathers color information related to surfaces inside their FOV, a 3-dimensional scanner records distance information regarding surfaces inside its FOV. The "picture" generated by a 3-dimensional scanner details the distance to a surface at every point in the picture. This lets the $3 \mathrm{D}$ position of each one of point in the picture to be determined. There are different types of techniques for digitally obtaining the shape of a threedimensional object. A well-established category [4] splits them into two categories: contact 3dimensional scanner and non-contact 3-dimensional scanner. The Non-contact 3-dimensional scanners are also split into two main categories: passive and active scanners. There are different types of technologies that belong to each of these categories [5].

Laser scan is a non-invasive current method got large application in medical field. The conversions of the metrological knowledge of the impact to a 3D digital metrological knowledge can be achieved with laser methods able to achieve a 3D laser scanning of the original ear impression. 
In any case, the entire shell-making procedure is totally dependent upon the ear impression and, this can be the only reason for weak fitting shell. The aim of a 3D scanner is generally to produce a point cloud of geometrical samples over the surface of the object. These types of points $(\mathrm{x}, \mathrm{y}, \mathrm{z})$ will then be utilized to extrapolate the shape of the object and this procedure is known as reconstruction. If color details are obtained at each point, then these colors (R, G, B) on the object surface can be calculated. 3D scanners are very similar to cameras. As cameras, they contain a cone-like field of view, and also will only obtain details about surfaces that aren't hidden. While camera gathers color details about surfaces throughout their field of view, 3D scanners gather distance details of surfaces throughout its field of view. The "picture" created by a 3D scanner identifies the distance to a surface at every point in the image [6]. This work is aiming to propose a 3D scanner for external with low cost and good accuracy. In [7] authors reported on the technique of rapid production of hearing aids with rapid shell modeling (RSM) computer aided design and manufacturing (CADCAM).

\section{Previous works}

In 2004, Tognola et al. [8] presented a method for hearing aid shells design by 3D laser scanning along with mesh reconstruction, the suggested system is using a couple of cameras and a retail laser for the surface digitization and so on a straight forward algorithm, depending on the deformation of a geometric model, for acquired surface reconstruction. The measurements on objects dimensions and features are implemented to evaluate the repeatability levels and the accuracy of this three-dimensional acquisition system. Furthermore, they determined the robustness to noise of the presented reconstruction algorithm from simulations using a synthetic test surface. At last, the initial measurement (reconstruction and acquisition) of closed surfaces from ear canal impressions has been reported.

In 2016, Ali et al. [9] employed a laser method of size and shape reconstruction of hearing aids. The proposed system is made up of: a laser source (Green) of 532nm wavelength, and with power about $(51 \mathrm{mw})$ with reflector of $(1 \mathrm{~mm})$ thickness. Otoscope is made with stainless steel type (302). They used MATLAB software for calculating human ear canal dimensions by utilizing algorithm. A clear human ear canal image has been taken by using the proposed system. Even so, the ear canal dimensions are measured choosing the average time about (10 minutes). The Percentage error (P.e.) of the ear canal length is $( \pm 0.72 \%)$, and P.e. of ear canal width is $( \pm 1.33 \%)$. At last, the results are viewed as a proofof-concept to calculate the human ear canal dimensions automatically for hearing aid production.

In 2016 Chiou et al. [10] study the 3D surface anthropometry of ear canal, the method involve that the ear impression had been obtained by injection silicon rubber then the portable three dimensional camera has been used to gather three dimensional auditory canal data from the ear impression models. The study results prove that the main factor for external auditory canal dimensions for hearing protection are often the canal isthmus, the ear aperture, the length of from ear aperture to first and second bend and the depth, width and length of concha, as well as, the variations in size of ear canal between women and men.

\section{Theoretical background}

The most important goal of three- dimensional (3D) imaging is to present both qualitative and quantitative information with regards to a system or object from images acquired with multiple modalities such as: laser technique, SPECT, CT, MRI, and PET [11]. There are different types of techniques for digitally obtaining the shape of a three-dimensional object. A well-established category [12] splits them into two categories: contact 3-dimensional scanner and noncontact 3-dimensional scanner. The Non-contact 3dimensional scanners are also split into two main categories: passive and active scanners. There are different types of technologies that belong to each of these categories [13].

\subsection{Contact technique}

It is the metrological system that may give the coordinate of the object to be measured easily via touching it with a laser or optical probe. A common type of this method is Coordinate Measuring Machine (CMM) that generally has 3 or more axes. Every one of axis has their measuring scale for feedback and position control. Once the probe tip touches a component surface, a little deflection of the probe is noticed that triggers an operation of checking off the present position of the contact point over the 3 axes. The readings can be easily transformed into 3D coordinates of the centre of the probe depending on the configuration of the CMM. Hence, a single point to the part surface is measured and then the process will be repeated $[14,15]$. 
3.2Non-contact technique

This technique can be divided to two main categories: passive and active. Passive scanners don't emit any type of the radiation; however, it depends on detecting reflected background radiation. This type of 3D Laser Scanners can typically have categorized into three typical categories [16].

1. Laser Triangulation: Laser triangulation is a process to measure the absolute distance for an object. The concept of triangulation is provided including the extensions to $2 \mathrm{D}$ and $3 \mathrm{D}$ measurements.

2. Time of Flight (TOF): The Time-of-Flight (ToF) devices as well as Light Detection and Ranging (LIDAR) devices run using the fundamental of the Radio Detection and Ranging (RADAR) theory that depends on the concept that the electromagnetic radiation moves in air with a light speed.

3. Phase Shift: Phase shift scanners utilize a laser light whose intensity is sinusoidal modulated with time. From the reflected signal, a phase difference emitted the round-trip distance could be calculated, since the difference is proportional to the travelled distance.

\subsection{Microsoft Kinect camera as a $3 \mathrm{D}$ measuring device}

Kinect is the device containing a near-infrared (NIR) laser pattern projector, an infrared (IR) and color camera. The IR projector and camera utilized like a stereo set to triangulate points in three-dimensional space. The RGB camera is usually used to recognize the image content or texture the $3 \mathrm{D}$ points. As a measuring device, Kinect provides three outputs: RGB image, IR image, and a depth image (inverse) [17].

\section{Proposed 3D ear scanner design}

The system is based on used Microsoft Kinect professional version to reconstruction the external ear. It has been connected to computer in order to streaming video and depth map in order to preprocessing by ear scanner program to reconstruction external ear with real dimensions.

\subsection{System setup}

Kinect for windows version 1 has been used for this work [18]. The Kinect has RGB camera with resolution up to $(640 \times 480$ pixels @ $30 \mathrm{~Hz})$ and IR depth-finding camera have a resolution up to (640×480 pixels@30 Hz). Figure 1(a) show the Kinect version 1 used in system. The Kinect has been mounted on a handle arm in order to create a 3D ear scanner, as shown in Figure 1(b).

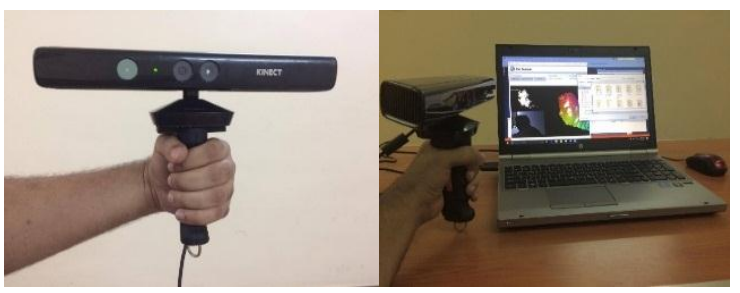

(a)

(b)

Figure 1 Proposed 3D ear scanner, Kinect has been mounted on handled arm in order to easy carry and scanning ear and it had been connected to laptop via USB

\subsection{Ear reconstruction program}

The external ear reconstruction algorithm is based on point cloud library (KinFu). It got point cloud from image depth that recorded by Kinect through time then generate poly mesh on these points. KinFu was the earliest open-source application of Kinect Fusion that available to anyone. It had been designed as part of the point cloud library (PCL) in 2011, It has a GPU implementation of the Marching Cubes algorithm which calculates a triangle mesh rather than a point cloud. The point cloud library is a group effort, with a few of the code added as one-off lacking and contributions thorough review and active maintenance. $\mathrm{KinFu}$ is the best feature-complete Kinect Fusion application available, and several corresponding projects are designed upon it [19].

The external ear reconstruction algorithm described in follows:

\section{External algorithm}

Input: Depth Map from Kinect.

Output: Arabic Stego Text.

Step 1: Start

Step 2: Conversion of the Depth map. This step gets the raw depth from the Kinect and changes it to the floating-point depth in meters, then, it followed by an optional conversion for an oriented point cloud that provides 3D points/vertices within the coordinate system of camera, and also the surface normal (surface orientation) at those points to be used with the Align Point Clouds function.

Step 3: Global/world camera pose calculation. This step will calculate the location and orientation of camera pose and tracks that pose as the sensor passes in every frame utilizing an iterative alignment algorithm, therefore the system often knows the present sensor pose in accordance with the initial 
starting frame. The iterative alignment algorithm algorithms could be used to arrange point clouds computed to reconstruction by using the new incoming point clouds that come from camera depth of Kinect.

Step 4: The depth data Integration. This step will integrate the depth data from the referenced sensor pose towards a single volumetric representation at area surrounding the camera. This integrated of the depth information is taken out per-frame, continually, using a running average to minimize noise, however handle a few dynamic changes in the view (for example, the small objects to be added or removed). When moving sensor views a surface through a little different viewpoint, any holes or gaps in which depth data could be not present in, the actual Kinect image will also be filled in (i.e. it can fill the rear of the object by moving the sensor over an object) as well as the surfaces are frequently refined with the new high-resolution data when the camera approaches the surface much closely.

Step 5: Volume Reconstruction. This is the final step that can be ray-cast from the sensor pose (that's usually, but is not limited to the present Kinect sensor pose), where the resulting point cloud could be shaded for a rendered visible 3D reconstruction volume image.

Step 6: End.

\section{Results}

This section shows the experiments results that lead to measure the performance of the proposed system. The 3D ear scanner software has software has been developed by using visual studio 2017 with C\# language. The tested has been run by used a laptop (HP) with following specifications:

- CPU: 2.4 core i7

- 8GB DDR3 RAM

- Operation system: Windows 10 64bit

- Visual studio 2017

\subsection{External Ear reconstruction program} parameters

In our test, we scanned real human ear with handled option in order to reconstructed ear in real time. Figure 2 shows the experimental test on human face in order to reconstructed external ear.

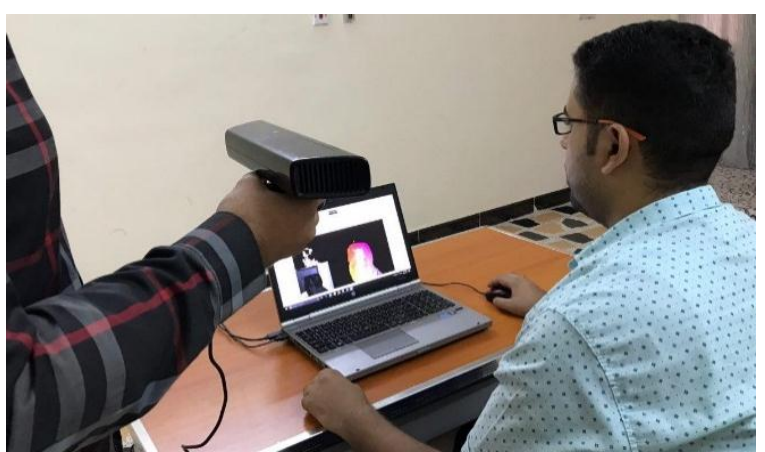

Figure 2 The human face scanned in order to reconstruct external ear

In order to select the optimal setting that can be used for ear scanning in room condition we test several parameters that effect on ear reconstruction that used by KinFu library. The most effective parameter is the integration weight and the removing background.

The removing background is done be determine the maximum depth parameter is the distance threshold in meters. Depth pixels above this value will be returned as invalid (0). Max depth must be greater than 0 . This parameter is very important to separation human face from background. The proper value determines manually depending on place and environment that can user see the result by sliding the background remover slider until getting the required result. Figure 3 illustrates the separation of human face from background with different values.

The integration weigh is effect on surface reconstruction with moving camera. It re calculate generated structure with each new coordinate in order to integrate the new result with previous structure to builds the continues structure. This very important to reconstructed bodies in 3D with 360 degrees (front view, sides and the rear).

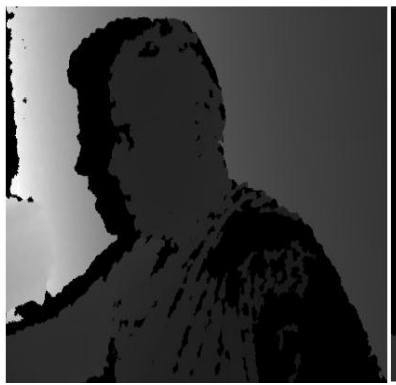

(a)

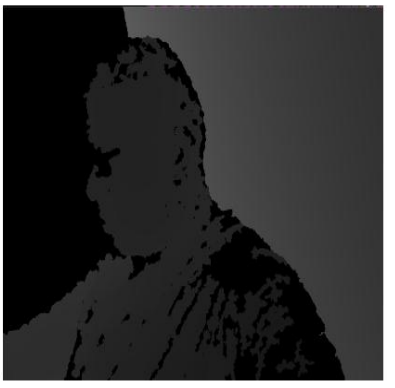

(b) 


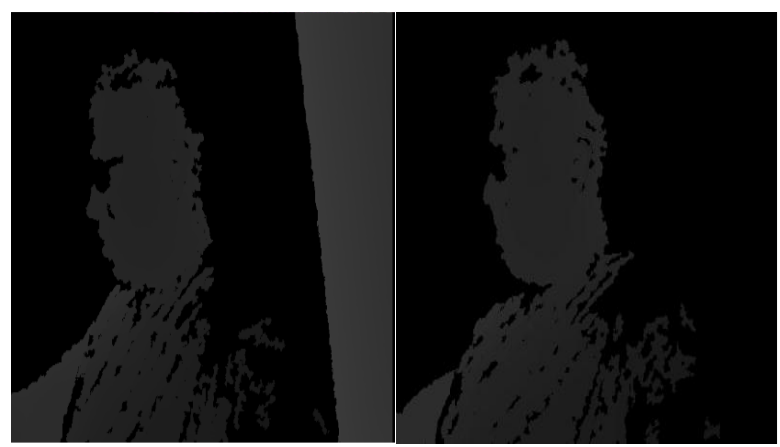

(c)

(d)

Figure 3 The removing background results with values (a), (b), (c) and (d)

The higher value makes integration fast and low makes it slow. The proper value also should determine manually by user depending on place the hand scanning speed and environment, where user can see the result in real time by sliding the integration value slide until getting the required result. Figure 4 illustrates the reconstruction results with integration value with handled scanner.

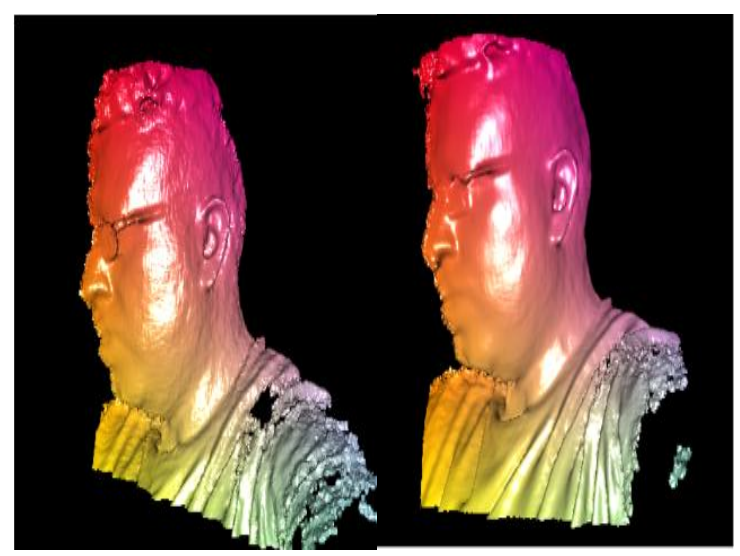

(a)

(b)

Figure 4 (a) The user interface window of external ear reconstruction program with integration value is equal to 500 (b) the integration value is 1000

Depending on scanning environment, we have been determining the proper values of the integration weight and the removing background to be 699 and 9 respectively. The scanning result shows in Figure 5.

The operation is done by taking the image depth from Kinect with a lot of lost data and then creating a realistic smooth Three-dimensional reconstruction of the static scene through moving the Kinect sensor around. From this, a point cloud then a 3D mesh will be produced.

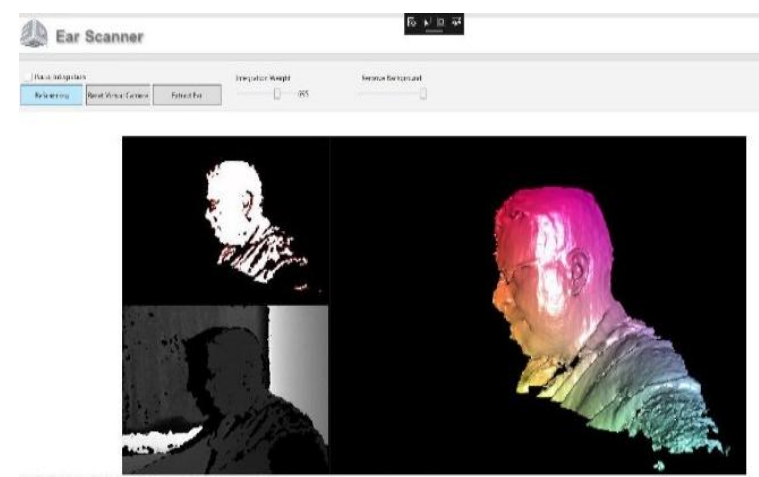

Figure 5 The human face scanned result where: lower left: the depth image, upper left: the field of view of point clod and reconstruction mesh, the right: is the scanning results

The system reconstructs only one dense surface model along with smooth surfaces via integration the depth data from Kinect with time from several points of views. The camera pose will be tracked while the sensor is moved and it will be able to recognize every frame's pose and how it requires the rest, these kinds of multiple viewpoints of the environment or objects could be fused together to be a single reconstruction voxel volume. The result has high details when compared to real ear detail as shown in Figure 6.

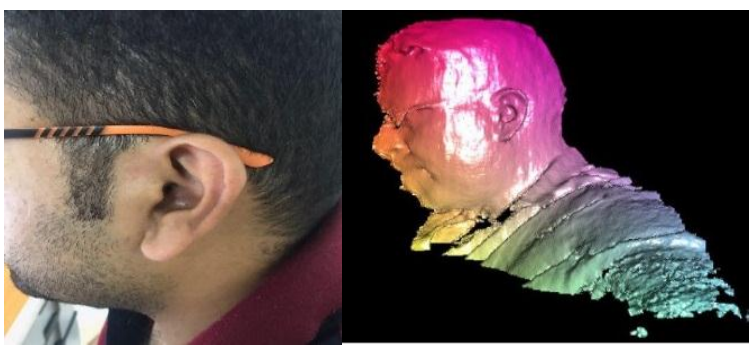

(a)

(b)

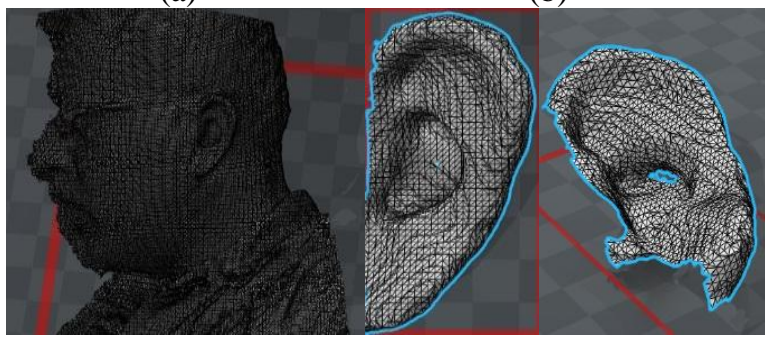

(c)

(d)

(e)

Figure 6 (a)Real human face (b)reconstruction result (c)3D humane reconstruction object (d \& e) 3D ear object result

\section{Conclusion and future work}

Three-dimensional reconstruction is a highly beneficial method of object creation using a photo- 
realistic way especially in medical applications. For 3D ear scanner application (such as hearing aid), the accuracy in ear details and measurement is the main issue that should be put in consideration, also the mobility and ease of use is the second issue. This paper has presented the theoretical and experimental approach for design a low cost handled 3D scanner for external ear. The proposed system is based on utilized Microsoft Kinect with reconstruction program. The system consists of Microsoft the Kinect version 1 with proposed software based on KinectFusion in order to reconstruct external ear in real-time.

From the results of reconstruction and error analysis we pointed the following:

1. To eliminate or minimize the distortions in the point cloud and to getting a good detail and avoid misalignments between the depths and color data an accurate stereo setting of the IR and the RGB camera is very important to getting the desired result.

2. The random error of measuring the depth will be increases quadratically as distance between scanned object and sensor has increasing. Also the depth resolution will reduce quadratically with increasing distance from the sensor. To avoid that, the scanning range and field of view has been determined in order to obtain a close result to real measurement. The system shows no results when the human head above the appropriate distance.

The results show high accuracy of ear details that related to reconstruction algorithm and reconstruction software that yield good 3D meshes of the external ear.

\section{Acknowledgment}

My sincere thanks to my supervisor the source of knowledge and inspiration Prof. Dr. Ziad Tarik AL-Dahan for his suggestion advice, support and help in the project to complete this work. I would also like to express my sincere gratitude and appreciation to the head of the department Dr. Sadiq for all the support and guidance provided throughout my education, and to the other teaching members in the department.

\section{Conflicts of interest}

The authors have no conflicts of interest to declare.

\section{References}

[1] Kalliany R. Photogrammetric computer vision: proceedings of the ISPRS Commission III symposium. Institute for Computer Graphics and Vision, Graz University of Technology; 2002
[2] Nixon MS, Aguado AS. Feature extraction \& image processing for computer vision. Academic Press; 2012.

[3] Udupa JK. Three-dimensional visualization and analysis methodologies: a current perspective. Radiographics. 1999; 19(3):783-806.

[4] Curless B. From range scans to 3D models. ACM SIGGRAPH Computer Graphics. 1999; 33(4):38-41.

[5] Ebrahim M A. 3D Laser scanners' techniques overview. International Journal of Science and Research. 2015; 4(10):323-31.

[6] Rakitina E, Rakitin I, Staleva V, Arnaoutoglou F, Koutsoudis A, Pavlidis G. An overview of 3D laser scanning technology. In proceedings of the international scientific conference. 2008.

[7] Gao T, Jarng SS. Design and realization of hearing aids based 3D rapid shell molding CADCAM. In international symposium on industrial electronics. 2009 (pp. 1488-92). IEEE.

[8] Tognola G, Parazzini M, Svelto C, Galli M, Ravazzani P, Grandori F. Design of hearing aid shells by three dimensional laser scanning and mesh reconstruction. Journal of Biomedical Optics. 2004; 9(4):835-43.

[9] Ali NM, Al-Dahan ZT, Rashid FF, Mahmood RS. Laser technique for shape and size reconstruction of hearing aids for the human ear canal. Journal of Nanoscience and Technology. 2016: 176-7.

[10] Chiou WK, Huang DH, Chen BH. Anthropometric measurements of the external auditory canal for hearing protection earplug. In advances in safety management and human factors 2016 (pp. 163-71). Springer International Publishing.

[11] Rutka J. Discussion paper on hearing loss. http://www.vrab-tacra.gc.ca/Publications/DiscussionPaper-on-Hearing-Loss.pdf. Accessed 01 December 2017.

[12] WHO, Deafness and hearing loss. http://www.who.int/mediacentre/factsheets/fs300/en/. Accessed 01 December 2017.

[13] Prasciolu M. 3D laser scanner based on surface silicon micromachining techniques for shape and size reconstruction of the human ear canal. University of Trieste. 2007.

[14] Franceschini F, Galetto M, Maisano D, Mastrogiacomo L, Pralio B. Distributed large-scale dimensional metrology: new insights. Springer Science \& Business Media; 2011.

[15] Venuvinod PK, Ma W. Rapid prototyping: laser-based and other technologies. Springer Science \& Business Media; 2013.

[16] Faxin Y, Lu Z, Luo H, Wang P. Three-dimensional model analysis and processing. Springer-Verlag Berlin Heidelberg; 2011.

[17] Fossati A, Gall J, Grabner H, Ren H, Konolige K.Consumer Depth Cameras for Computer Vision. Springer-Verlag Berlin Heidelberg; 2013.

[18] Jana A. Kinect for windows SDK programming guide. Packt Publishing LTD; 2012.

[19] Hambüchen N. HouseScan: building-scale interior 3D reconstruction with Kinect Fusion. 2014. 
Mohammad H. Basheet et al.

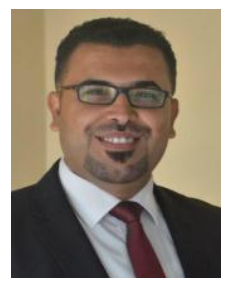

Mohammad H. Basheet is a master degree candidate and resercher at AlNahrain University, Baghdad, Iraq in the Depatment of Biomedical Engineering. He completed his bacheloar degree in Biomedical Engineering from the Hashimite University in Amman, Jordan. His research areas are Medical Imaging and Three Dimentional Laser Imaging.

Email: eng.moh1988@gmail.com

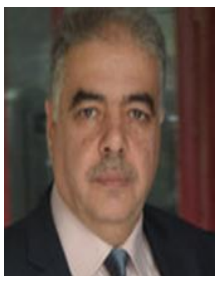

Ziad T. Al-dahan is a professor in the department of Biomedical Engineering at Al-Nahrain University, Baghdad, Iraq. His research areas are Material Characterization, Thin Films and Nanotechnology, Thin Film Deposition Optics Electrical Engineering, Semiconductor Device, Physics Laser, Nanoelectronics Electronic Engineering Biomedical Engineering, Sintering Microelectronics and Semiconductor Engineering, Microelectronics Detectors Absorption ,Conductivity of Silicon, Electrical Conductivity, XPS, Oxides Super conductors, Microelectronics Engineering, DTA ,Semiconductor Engineering, Zinc Oxide ,Photoelectron Spectroscopy Microelectromechanical Systems (MEMS) Vacuum Semiconductor Lase. 